\title{
WEAK SUBNORMALITY OF INFINITE 4-BANDED MATRICES
}

\author{
In Sung Hwang, In Hyoun Kim And Sumin Kim
}

Abstract. In this paper, we consider a class of operators whose matrix representations comprise 4-banded matrices, i.e., sparse matrices whose non-zero entries are confined to four diagonals. In particular, we focus on the hyponormality and weak subnormality when each diagonal forms a hyponormal weighted shift.

Mathematics subject classification (2010): 47B20, 47B37, 47A63.

Keywords and phrases: weak subnormality, hyponormality, 4-banded matrices, weighted shifts.

\section{REFERENCES}

[1] C. Cowen And J. Long, Some subnormal Toeplitz operators, J. Reine Angew. Math. 351 (1984), 216-220

[2] R. E. CURTO, I. B. JUnG, AND S. S. PARK, A characterization of k-hyponormality via weak subnormality, J. Math. Anal. Appl. 279 (2) (2003), 556-568.

[3] R. E. CURTO, S. H. LEE, AND W. Y. LEE, A new criterion for $k$-hyponormality via weak subnormality, Proc. Amer. Math. Soc. 133 (6) (2004), 1805-1816.

[4] R. E. CURTO AND W. Y. LEE, Towards a model theory for 2-hyponormal operators, Integral Equations Operator Theory 44 (2002), 290-315.

[5] C. GU, I. S. Hwang AND W. Y. LeE, Hyponormality of Toeplitz operators in several variables by the weighted shifts approach, Linear and Multilinear Algebra 68 (8) (2020), 1695-1720.

[6] I. S. HwANG, Hyponormality of Toeplitz operators on the Bergman space, J. Korean Math. Soc. 45 (2008), 1027-1041.

[7] J. LEE, Hyponormality of Toeplitz operators on the weighted Bergman space with matrix-valued circulant symbols, Linear Algebra Appl. 576 (2019), 35-50. 